\title{
Intractable Hiccups due to Isolated Parainfectious Myelitis
}

\author{
Samhita Panda ${ }^{1}$ Diwakar Verma ${ }^{1}$ \\ ${ }^{1}$ Department of Neurology, All India Institute of Medical Sciences, \\ Jodhpur, Rajasthan, India
}

\begin{abstract}
Address for correspondence Samhita Panda, DM, Department of Neurology, All India Institute of Medical Sciences, Jodhpur 342001, Rajsthan, India (e-mail: samhitapanda@yahoo.com).
\end{abstract}

\begin{abstract}
Keywords

- intractable hiccups

- demyelination

- myelitis

- neuromyelitis optica
\end{abstract}

\section{Introduction}

Recurrent nausea and vomiting is a frequent symptom generally dealt with in general medicine, gastroenterology, and surgical outpatient clinics or emergency. There are several causes ranging from infection to chronic medical conditions. ${ }^{1}$ At times, raised intracranial pressure can cause vomiting. At times, there can be hiccups which can be persistent due to a repeated diaphragmatic contraction paralleling a sudden glottic closure. ${ }^{2}$ The centers controlling hiccups and vomiting are located in medulla oblongata. ${ }^{3}$ Intractable hiccups, which last for more than 48 hours, may sometimes be the only presenting feature of demyelinating disorders such as neuromyelitis optica (NMO). Reports on intractable hiccups in demyelinating disorders without brainstem lesion are rare. An unusual case of isolated myelitis presenting with intractable hiccups is reported.

\section{Case Report}

A 33-year-old male patient presented with complaints of low-grade fever for 6 to 7 days, recurrent vomiting for 4 to 5 days, and persistent hiccups since 4 days. He was also having unsteadiness of gait and decreased sleep, primarily due to incessant hiccups. There was no associated headache, seizure, difficulty in vision, hearing, chewing, swallowing, or limb weakness and no sphincteric complaints. There was no history of dyspepsia, malabsorption, chest pain, or chest discomfort. There were no significant comorbidities, cardiovascular, cerebrovascular, or similar illness in the past. He was chronic smoker for 4 years.

At presentation, general physical and systemic examination was unremarkable. Nervous system evaluation was normal except mild gait ataxia. Hematological and biochemical tests including complete blood count, liver function, kidney function tests, and serum electrolytes were normal. Peripheral blood smear for malarial parasite and serology for dengue was negative. Serology for HIV and Hepatitis B and C were negative. Electrocardiography (ECG) was normal. Mantoux test after 48 hours was negative. Cerebrospinal fluid (CSF) examination showed normal sugar $(45 \mathrm{mg} / \mathrm{dL})$, elevated protein level (181 mg/dL), and cells (500/cumm) which were predominantly lymphocytes. CSF was negative for India ink, Gram's stain, Ziehl-Neelson stain, and aerobic cultures. CSF for TB PCR (polymerase chain reaction for mycobacteria in tuberculosis) and CSF aquaporin antibody was also negative. Electrophysiological studies including visual evoked potentials and brainstem auditory evoked potentials were normal. Multiplanar 
images of brain and whole spine were acquired using GE discovery $750 \mathrm{~W}$ 3T magnetic resonance imaging (MRI). MRI of brain was normal, while MRI of spine showed long segment T2 hyperintensity in dorsal spinal cord extending up to conus medullaris without any cord expansion (-Fig. 1).

At the onset the hiccups were treated empirically with metoclopramide, ondansetron, baclofen, clonazepam, and antacids without any relief. As on MRI results, the possibility of myelitis of probable parainfectious etiology was considered, the patient was given injectable methylprednisolone $1 \mathrm{~g}$ intravenously for 5 days. Hiccups stopped on day 2 of methylprednisolone. The patient was subsequently discharged on short course of oral steroids. He remained asymptomatic on follow-up.

\section{Discussion}

Hiccups are caused by a repetitive and involuntary contraction of the diaphragm accompanied by sudden glottic closure. ${ }^{2}$ There are peripheral, as well as central, causes for vomiting and hiccups. Intractable hiccup is defined by hiccups and nausea lasting more than 48 hours, despite common therapeutic management. ${ }^{3}$ Apart from gastroenterological causes, intractable hiccups are commonly encountered in inflammatory demyelinating diseases such as NMO and rarely in multiple sclerosis. ${ }^{4}$ The present case highlights the rare occurrence of intractable hiccups in isolated myelitis.
Mechanism of hiccups in demyelinating disorders is unclear. The dorsomedial medulla oblongata (including area postrema, nucleus tractus solitarius, ventrolateral respiratory center, and nucleus ambiguous) have the hiccup and vomiting centers. In NMO, the primary inflammatory targets are the astrocytes, specifically in the area postrema, where the binding of immunoglobulin (Ig) G antibodies to astrocytic aquaporin AQP4 water channels result in nausea and vomiting. ${ }^{3,5,6}$ Very rarely, an isolated spinal cord lesion may stimulate the hiccups reflex arc activating the hiccup center and resulting in intractable hiccups. As per hiccup reflex arc suggested by Kahrilas and Shi, the vagus, phrenic, and sympathetic nerves of $\mathrm{T} 6$ to 10 are the afferents, while the phrenic and vagus nerves are the efferents. ${ }^{7}$ The reflex center is located at the anterior horns at C3 to 5 level and the medulla oblongata and further regulated by the cerebral cortex, limbic system, and the hypothalamus. Thus, the hiccup center may be activated by cervical cord lesions and be the mechanism in the present case.

The classical demyelinating disorder in which refractory vomiting and hiccups may rarely be the sole manifestation of the first attack, is NMO, which was first described by Eugene Devic in 1894..$^{89}$ Oversight may lead to the diagnosis being missed at this stage leading to delay in treatment and further complications. NMO spectrum disorder (NMOSD) is now recognized to be a relapsing disease rather than purely a monophasic disorder including atypical presentations,

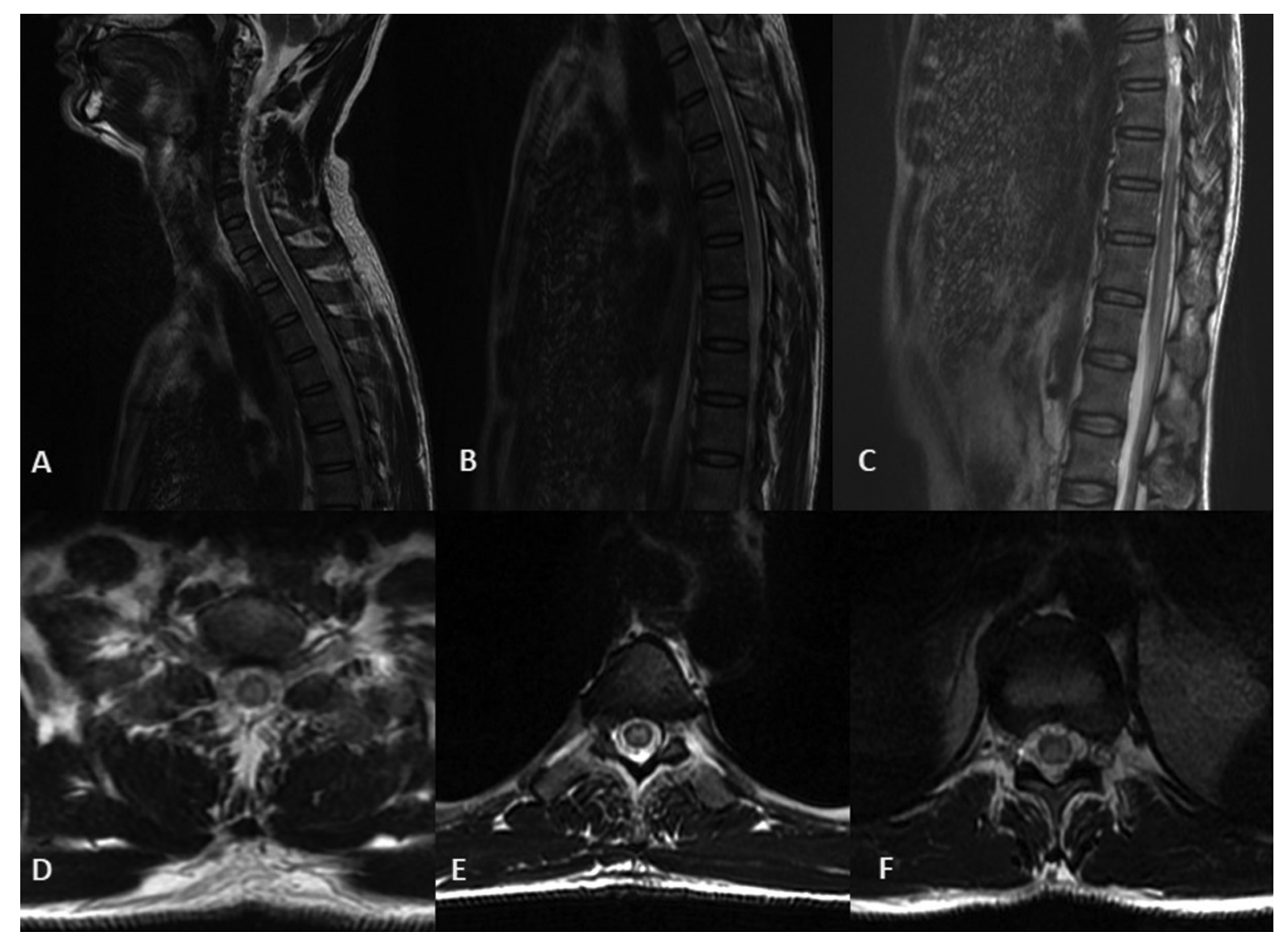

Fig. 1 T2-weighted sagittal (A-C) and axial (D-F) images of 3T MRI of spine at the level of cervicodorsal (A,C), dorsal (B,D), and dorsolumbar $(\mathbf{C}, \mathbf{F})$ regions demonstrating central cord hyperintensity extending from dorsal level to conus medullaris consistent with long segment myelitis. 
such as hypersomnia, intractable hiccups, nausea, vomiting, transient elevation of creatine kinase level, and painful tonic limb spasm. As per the diagnostic criteria for NMOSD, the core clinical presentations include the six neuroanatomic locations of optic nerve, spinal cord, area postrema (dorsal medulla), diencephalon, brainstem, and cerebrum. ${ }^{10}$ Therefore, a linear medullary MRI lesion in the area postrema may indicate the diagnosis of NMOSD even when the classical clinical criteria are not met, thereby necessitating aquaporin antibody testing. In the absence of aquaporin antibody, the diagnosis of NMOSD could not be considered in this patient as per the present guidelines.

An extensive review of literature has suggested only two reported cases of myelitis presenting with hiccups till date. ${ }^{11}$ Of six patients with cervical lesions without brain or medulla oblongata lesions having hiccups lasted from 2 to 23 days (average: $9.33 \pm 8.64$ days). Of these, one patient had acute myelitis and another recurrent myelitis with long segment involvement. The rest had NMO and multiple sclerosis. The duration of hiccups in these patients was 2 and 3 days, respectively, and both responded well to methylprednisolone with subsequent recurrence as noted in the present report.

In the case reported here, the diagnosis of isolated parainfectious myelitis was considered due to the monophasic presentation in the absence of involvement of other sites in the neuraxis, clinically and radiologically. The presence of fever and CSF pleocytosis also supported parainfectious myelitis. This clinicoradiological picture and absence of aquaporin antibodies presently differentiated from the NMOSD. However, the myelin oligodendrocyte glycoprotein (MOG) antibodies were not tested for due to lack of availability. Thus, the recently described entity of MOG-IgG-associated optic neuritis, encephalitis, and myelitis (MONEM) may exist in this patient. ${ }^{12}$ Long-term follow-up of the patient may further clarify the diagnosis.

\section{Conclusion}

Contrary to general belief, intractable hiccups can be associated with nonsurgical neurological conditions. Demyelinating disorders like NMOSD are an important cause of intractable hiccups and, at times, its sole manifestation. In absence of aquaporin antibodies, NMO could still be considered, though the mandated criteria were not met. This case demonstrates the rare situation of isolated parainfectious myelitis, currently not classifiable as NMO, presenting as intractable hiccups and subsequent immediate, and lasting response to immunosuppression.

\section{Funding}

None.

\section{Conflict of Interest}

None declared.

\section{References}

1 Enweluzo C, Yarra P. Neuromyelitis optica: an often forgotten cause of intractable nausea and vomiting. Case Rep Gastroenterol 2013;7(2):281-286

2 Kobayashi Z, Tsuchiya K, Uchihara T, et al. Intractable hiccup caused by medulla oblongata lesions: a study of an autopsy patient with possible neuromyelitis optica. J Neurol Sci 2009;285(1,2) :241-245

3 Misu T, Fujihara K, Nakashima I, Sato S, Itoyama Y. Intractable hiccup and nausea with periaqueductal lesions in neuromyelitis optica. Neurology 2005;65(9):1479-1482

4 Takahashi T, Miyazawa I, Misu T, et al. Intractable hiccup and nausea in neuromyelitis optica with anti-aquaporin-4 antibody: a herald of acute exacerbations. J Neurol Neurosurg Psychiatry 2008;79(9):1075-1078

5 Parratt JD, Prineas JW. Neuromyelitis optica: a demyelinating disease characterized by acute destruction and regeneration of perivascular astrocytes. Mult Scler 2010;16(10):1156-1172

6 Popescu BF, Lennon VA, Parisi JE, et al. Neuromyelitis optica unique area postrema lesions: nausea, vomiting, and pathogenic implications. Neurology 2011;76(14):1229-1237

7 Kahrilas PJ, Shi G. Why do we hiccup? Gut 1997;41(5):712-713

8 Miyazawa I, Fujihara K, Itoyama Y. Eugène Devic (1858-1930). J Neurol 2002;249(3):351-352

9 Kulkarni GB, Kallollimath P, Subasree R, Veerendrakumar M. Intractable vomiting and hiccups as the presenting symptom of neuromyelitis optica. Ann Indian Acad Neurol 2014;17(1):117-119

10 Wingerchuk DM, Banwell B, Bennett JL, et al; International Panel for NMO Diagnosis. International consensus diagnostic criteria for neuromyelitis optica spectrum disorders. Neurology 2015;85(2):177-189

11 Hao XT, Wang L, Yan B, Zhou HY. Intractable hiccup caused by spinal cord lesions in demyelination disease. J Spinal Cord Med 2013;36(6):711-714

12 Dos Passos GR, Oliveira LM, da Costa BK, et al. MOG-IgG-associated optic neuritis, encephalitis, and myelitis: lessons learned from neuromyelitis optica spectrum disorder. Front Neurol 2018;9:217 